\title{
Age-dependent tissue expression patterns of Sirt1 in senescence-accelerated mice
}

\author{
HUAN GONG，JING PANG，YIWEN HAN，YANG DAI，DAPENG DAI，JIANPING CAI and TIE-MEI ZHANG \\ Department of Cell Biology, The Key Laboratory of Geriatrics, Beijing Hospital and Beijing Institute of Geriatrics, \\ Ministry of Health, Dongcheng, Beijing 100730, P.R. China
}

Received October 16, 2013; Accepted June 5, 2014

DOI: $10.3892 / \mathrm{mmr} .2014 .2648$

\begin{abstract}
Sirtuin 1 (Sirt1) has a range of molecular functions and has emerged as an important protein in aging and metabolic regulations. Studies have reported a correlation between disturbance of Sirtl activity and the onset of aging- or obesity-associated diseases, including diabetes, cardiovascular disease and neurodegenerative disorders. However, a systematic investigation to examine the changes of Sirt1 expression in a wide range of ages and to what degree it changes has yet to be performed. To assess the effects of aging on the changes of Sirt1 expression, an in vivo model of aging, senescence-accelerated mouse prone 8 (SAM-P8) and a control counterpart strain, senescence-accelerated mouse resistant 1 (SAM-R1) was used. The mRNA and protein expression levels of Sirtl were detected in four different tissues, including brain, liver, skeletal muscle and white adipose tissue, in both strains at different ages (1-, 4-, 8- and 12-month old). Therefore, different life stages, including young age prior to maturation, adult, middle age and old age were examined. The results identified that Sirtl expression declined with age at the transcriptional and translational levels in the brain, liver, skeletal muscle and white adipose tissue in SAM-P8 and SAM-R1. The Sirt1 expression level was lower in SAM-P8 than in SAM-R1, particularly at old age. Among the four tissues, it was most significantly reduced in the brain.
\end{abstract}

\section{Introduction}

Sir2-related proteins or sirtuins are highly conserved NAD(+) dependent deacetylases that have been demonstrated to regulate the lifespan of lower organisms $(1,2)$ and affect diseases associated with aging in mammals, including diabetes, inflammation and neurodegenerative disorders (3). The Sir2 ortholog Sirtuin 1 (Sirt1) functions as an intracellular energy

Correspondence to: Professor Tie-Mei Zhang, Department of Cell Biology, Beijing Hospital and Beijing Institute of Geriatrics, Ministry of Health, 1 Dahualu, Dongcheng, Beijing 100730, P.R. China

E-mail: tmzhang126@126.com

Key words: sirtuin 1, senescence-accelerated mouse, aging, brain, liver, skeletal muscle, white adipose tissues sensor to detect the concentration of $\mathrm{NAD}(+)$, and controls in vivo metabolic changes under caloric restriction and starvation through its deacetylase activity to numerous targets, including histones, nuclear transcriptional factors and enzymes important for aging and disease $(4,5)$. Sirt1 has a range of molecular functions and has emerged as an important protein in aging and metabolic regulation (6). During the past decade, studies have reported a correlation between Sirt1 activity and aging-associated diseases, including diabetes, cardiovascular disease and neurodegenerative disorders. Transgenic mice with 2-fold higher levels of Sirt1 expression globally are protected against metabolic decline due to aging (7-9). Sirt1 mutant mice were small and exhibited notable developmental defects of the retina and heart, and only infrequently survived postnatally $(10,11)$. However, systematic investigations reporting the association of changes in Sirt1 expression with age are lacking.

In vivo animal models facilitate investigations aimed at elucidating changes in Sirtl expression. The senescence-accelerated mouse (SAM) is an accelerated aging model that was established through phenotypic selection from a common genetic pool of the AKR/J strain of mice (12). The SAM model was established in 1981, including nine major senescenceaccelerated prone, short-lived mice (SAM-P) substrains and three major senescence-accelerated resistant, long-lived mice (SAM-R) substrains. In the SAM-P strains, normal development and maturity of reproductive function are observed, and the values for numerous (but not all) physiological and pathological parameters are similar to those in SAM-R strains at a young to mature age. The SAM-P strains grow normally, but then they exhibit early signs of senescence, including reduction in physical activity and skin quality, hair loss, periophthalmic lesions and increased lordokyphosis. These characteristic pathological phenotypes are similar to those observed in elderly humans. The life span of the SAM-P strains is 26\% shorter than that of the SAM-R strains. The common aging characteristic of SAM-P strains is senescence acceleration following normal development and maturation.

To assess the effects of aging on the changes of Sirt1 expression, an in vivo model of aging, SAM-P8, and a control counterpart strain, SAM-R1, were used. The life span of SAM-P8 mice ranges from 10-17 months, which is shorter than that of SAM-R1, which ranges from 19-21 months (13). The mRNA and protein levels of Sirt1 were detected in four different tissues, including brain, liver, skeletal muscle and white adipose tissue 
in both strains at different ages (1-, 4-, 8- and 12-month old), thus covering various different life stages, including young age prior to maturation, adult, middle age and old age. The results indicated that Sirt1 expression progressively decreased with age.

\section{Materials and methods}

Animals. Male SAM-R1 and SAM-P8 at 1-, 4-, 8- and 12-months old were donated by Professor Jianping Cai (Department of Molecular Biology, The Key Laboratory of Geriatrics, Beijing Hospital and Beijing Institute of Geriatrics, Ministry of Health, Beijing, China). All the animals were housed at $21^{\circ} \mathrm{C}$ in a $12 \mathrm{~h} \mathrm{light/dark} \mathrm{cycle.} \mathrm{The} \mathrm{chow} \mathrm{and} \mathrm{water}$ were provided ad libitum. The animals were allowed to acclimatize for 1 week. The rats were then weighed prior to sacrifice and the food intake over this time was calculated according to the formula: Weight of the initial chow (Wi) - weight of the leftover chow (Wl) - weight of the spilled chow (Ws)/ number of mice per cage/days. All the mice were sacrificed by cervical dislocation. The tissues were dissected on ice, washed with ice-cold phosphate-buffered saline (PBS), snap frozen in liquid nitrogen, ground into powder with mortar and pestle in liquid nitrogen and then stored at $-80^{\circ} \mathrm{C}$.

This study was approved by the Biomedical Ethics Committee of Beijing Hospital and Beijing Institute of Geriatrics, Ministry of Health. The National Institutes of Health (Bethesda, MD, USA) Guidelines for the Care and Use of Laboratory Animals were strictly followed and all of the experiments were approved by the Biomedical Ethics Committee of Peking University (Beijing, China).

RNA extraction and quantitative polymerase chain reaction ( $q P C R)$. Total RNA was isolated from 2-50 mg tissues using TRIzol $^{\circledR}$ reagent (Invitrogen Life Technologies, Carlsbad, CA, USA) following the manufacturer's instructions. First-strand cDNA was synthesized from $2 \mu \mathrm{g}$ of total RNA with random hexamer oligonuclueotide primers using a $20 \mu$ l reverse transcription system (New England Biolabs, Ipswich, MA, USA). A total of $1 \mu \mathrm{l}$ of cDNA was amplified by qPCR (7500; Applied Biosystems, Foster City, CA, USA) with SYBR-Green (Takara Bio, Inc., Shiga, Japan). HPRT was used as internal control to normalize the amplification result and the expression level of the SAM-R1 1-month-old group was normalized as ' 1 '. The primers for each PCR are as follows: Sirt1-US, CAGTGTCATGGTTCCTTTGC and Sirt1-DS, CACCGAGGAACTACCTGAT; HPRT-US, TGAC ACTGGCAAA ACAATGCA and HPRT-DS, GGTCCTTTTCA CCAGCAAGCT.

Protein extraction and western blot analysis. Approximately $50 \mathrm{mg}$ of the tissues were lysed in RIPA buffer supplied with $5 \mathrm{mM}$ ethylenediaminetetraacetic acid (EDTA), $1 \mathrm{mM}$ PMSF and complete protease inhibitor cocktail (Sigma, St. Louis, MO, USA). Following a brief sonication and incubation for $30 \mathrm{~min}$ on ice, the lysate was centrifuged at $14,000 \mathrm{x}$ g at $4^{\circ} \mathrm{C}$ for $15 \mathrm{~min}$ and the supernatant was used as a whole cell extract. The protein concentration was determined with a BCA protein assay kit (Pierce Biotechnology, Inc., Rockford, IL, USA) using BSA as a standard. The proteins in the sample buffer were denatured by maintaining them at $100^{\circ} \mathrm{C}$ for $3-5 \mathrm{~min}$. A total of $30 \mu \mathrm{g}$ of the total proteins were separated on a $12 \%(\mathrm{~W} / \mathrm{V})$ sodium dodecyl sulfate-polyacrylamide gel (SDS-PAGE) and transferred onto a PVDF membrane (EMD Millipore, Billerica, MA, USA). After blocking the membrane with 5\% (W/V) non-fat milk in Tris-buffered saline Tween-20 (TBST), the membrane was incubated overnight with primary antibody, washed in TBST, incubated with horseradish peroxidase (HRP) conjugated secondary antibody (anti-rabbit IgG from Sigma; anti-mouse IgG from Upstate Biotechnology, Lake Placid, NY, USA) at a dilution of 1:5,000 in TBST, washed and developed with ECL (Pierce Biotechnology, Inc.). The following primary antibodies were used: Sirt1 (05-707; Upstate Biotechnology), $\alpha$-tubulin (ab7291; Abcam, Cambridge, MA, USA) and $\beta$-actin (A5441; Sigma).

Statistical analysis. Statistical analyses were performed using SPSS statistical software. One-way analysis of variance (ANOVA) was used. Data are expressed as the group mean \pm standard error of the mean (SEM). $\mathrm{P}<0.05$ was considered to indicate a statistically significant difference.

\section{Results}

Changes in body weight of SAM-P8 and SAM-R1 with age. To assess the effects of aging on the changes of Sirt1 expression, four different age groups of both SAM-R1 and SAM-P8 were established; 1-, 4-, 8- and 12-months old, thus covering various different life stages, including young age prior to maturation, adult, middle age and old age.

Body weight (Fig. 1A) and appearance did not differ between the SAM-R1 and SAM-P8 at 1-month old (21.5 \pm 0.52 and $20.4 \pm 0.45 \mathrm{~g}$, respectively). However, the body weights of SAM-P8 were $8.7 \%(\mathrm{P}<0.05), 8.4 \% \quad(\mathrm{P}<0.05)$ and $11.0 \%(\mathrm{P}<0.05)$ lower than SAM-R1 in the 4-, 8 - and 12-month old groups, respectively (Fig. 1A). These data indicated that following developmental maturation, the SAM-P8 mice had a higher reduction in body weight than that of the SAM-R1 mice (Fig. 1A). Of note, there were no significant differences in the amount of food intake between the two strains at the different ages (Fig. 1B). These results suggested that the amount of food intake is not or at least not the only reason for the differences in body weight between the different ages and strains.

Sirtl expression pattern in adult SAM-RI tissues. To attempt to elucidate the expression pattern of Sirt1 in different tissues of normal adult mice, the protein level of Sirt1 in 12 tissues from 4-month old SAM-R1 rats were investigated (Fig. 1C). Among these 12 tissues, the testis, spleen, white adipose tissue and lung had the highest expression level, while pancreas, thymus, liver and brain had moderate expression levels, and in the skeletal muscle and intestine, the signals were extremely weak. The expression of Sirt1 in the kidney and heart was too low to be detectable.

Age-dependent changes in Sirtl mRNA expression level in different tissues of SAM. Then, the changes of Sirt1 expression patterns with age were investigated in the different tissues, including the liver, skeletal muscle, white adipose tissue and brain. These tissues cover high (white adipose tissue), moderate 
A

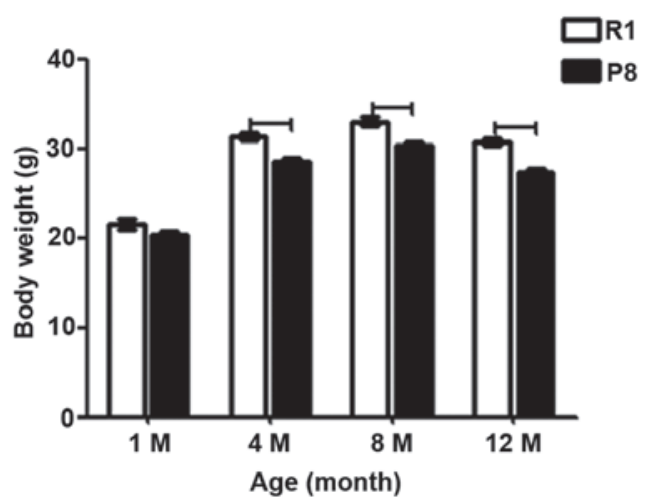

B

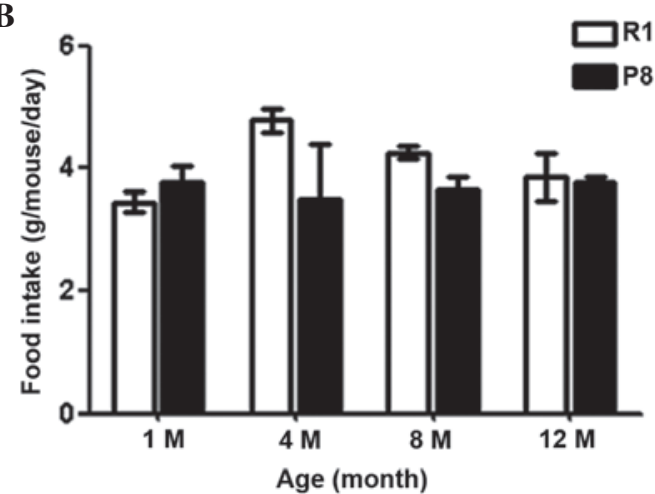

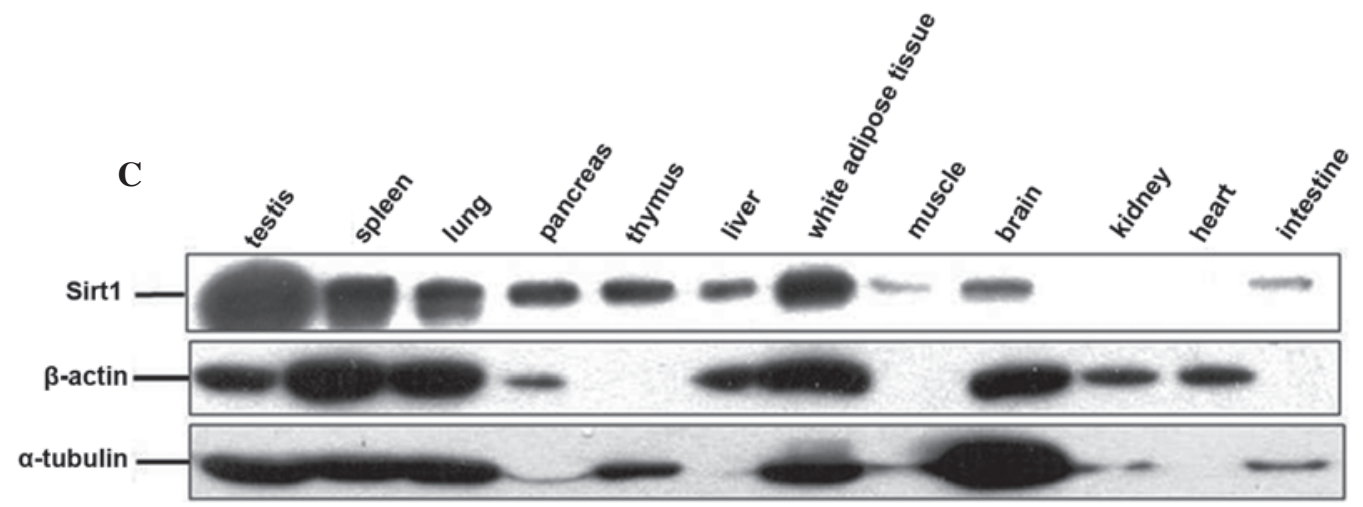

Figure 1. Basic information of SAM. The changes in (A) body weight and (B) food intake of SAM-R1 and SAM-P8 with age are expressed as the mean \pm SEM. R1, SAM-R1; P8, SAM-P8; 1M, 4M, 8M and 12M, 1-, 4-, 8- and 12-month old groups, respectively. (C) Western blot analysis of Sirt1 (upper panel), $\beta$-actin (middle panel) and $\alpha$-tubulin (lower panel) expression in 4-month old SAM-R1 tissues: testis, spleen, lung, pancreas, thymus, liver, white adipose tissue, skeletal muscle, brain, kidney, heart and intestine. The dash indicates the difference is significant between the two groups $(\mathrm{P}<0.05)$. SAM, senescence-accelerated mouse; SAM-R1, senescence-accelerated mouse resistant 1; SAM-P8, senescence-accelerated mouse prone 8; Sirt1, sirtuin 1; SEM, standard error of the mean.

(liver and brain) and low (skeletal muscle) expression level in all of the 12 tissues scanned. Furthermore, the liver, white adipose tissue and skeletal muscle belong to the metabolic system and regulate energy storage and consumption, and the brain is part of the central nervous system and thus secretes metabolism-regulating hormones.

Sirt1 mRNA levels decreased with age in all four tissues in both SAM-R1 and SAM-P8 (Fig. 2). The hypothesized maximal difference was to be between SAM-R1 1-month and SAM-P8 12-month old groups. Compared with the SAM-R1 1-month old group, the Sirt1 mRNAs level in the SAM-P8 12 -month old group reduced to $31.7 \%(\mathrm{P}<0.01)$ in the brain (Fig. 2A), 38.3\% ( $\mathrm{P}<0.01)$ in the liver (Fig. 2B), 46.4\% in skeletal muscle (Fig. 2C) and $38.3 \%(\mathrm{P}=0.064)$ in white adipose tissue (Fig. 2D). These differences were enhanced compared with those observed between the 12-month and 1-month old groups within the same strain. In the brain tissue compared with 1-month old mice, the Sirt1 mRNA level of 12-month old mice decreased to $38.1 \%(\mathrm{P}<0.01)$ in SAM-R1 and $38.3 \%(\mathrm{P}<0.01)$ in SAM-P8 (Fig. 2A); in the liver, decreased to $52.0 \%(\mathrm{P}<0.01)$ in SAM-R1 and 48.8\% ( $\mathrm{P}<0.01)$ in SAM-P8 (Fig. 2B); in skeletal muscle, decreased to $55.7 \%$ in SAM-R1 and $46.5 \%$ in SAM-P8 (Fig. 2C) and in white adipose tissue, decreased to $45.4 \%$ in SAM-R1 and $47.5 \%$ in SAM-P8 (Fig. 2D).

The majority of the Sirt1 mRNA expression in SAM-P8 was lower than that in the SAM-R1 strain at the same age. The levels in SAM-P8 were 82.9, 98.9, 89.7 and 83.4\% of that in the SAM-R1 from 1- to 12-months old, respectively, in the brain
(Fig. 2A); 79.5, 80.1, 86.8 and 74.6\% in the liver (Fig. 2B); 81.6, $82.1,86.3$ and $85.5 \%$ in white adipose tissue (Fig. 2D); and 89.1 and $83.3 \%$ of SAM-R1 at 8-months and 12-months old, respectively, in the skeletal muscle (Fig. 2C).

In the brain tissues (Fig. 2A), in SAM-R1, the Sirt1 mRNA level reduced to $55.8 \%$ (4-month; $\mathrm{P}<0.01$ ), $45.0 \%$ (8-month; $\mathrm{P}<0.01)$ and 38.1\% (12-month; $\mathrm{P}<0.01)$ sequentially, compared with the 1-month old group; in SAM-P8, it reduced to $66.6 \%$ (4-month), 48.7\% (8-month; $\mathrm{P}<0.05$ ) and 38.3\% (12-month; $\mathrm{P}<0.01$ ). In the liver (Fig. 2B), compared with the 1-month old group, the Sirt1 mRNA level gradually declined to 95.6, $58.8 \%(\mathrm{P}<0.01)$ and $52.0 \%(\mathrm{P}<0.01)$ in SAM-R1, and to 96.3 , $64.2 \%(\mathrm{P}<0.01)$ and $48.8 \%(\mathrm{P}<0.01)$ in SAM-P8 at $4-, 8$ - and 12-month old, respectively. The differences between 4-month old group with older groups were also significant $(\mathrm{P}<0.01)$ in both strains. In the skeletal muscle (Fig. 2C), the mRNA level also gradually declined, as compared with the 1-month old group in both strains; in SAM-R1, to 88.4, 65.8 and $55.7 \%$ at 4-, 8- and 12-month old, respectively; and in SAM-P8, to 88.8, 58.8 and $46.5 \%$ at 4-, 8- and 12-month old, respectively. In white adipose tissue (Fig. 2D), compared with the 1-month old group, in SAM-R1, the expression level decreased to $84.7 \%$ (4-month), 59.8\% (8-month) and 45.4\% (12-month), sequentially; in SAM-P8, decreased to $85.2 \%$ (4-month), $63.2 \%$ (8-month) and $47.5 \%$ (12-month) sequentially.

These data indicated that the Sirt1 mRNA expression level decreased with age in all four tissues in both strains, and the most marked reduction was detected in the brain. 
A

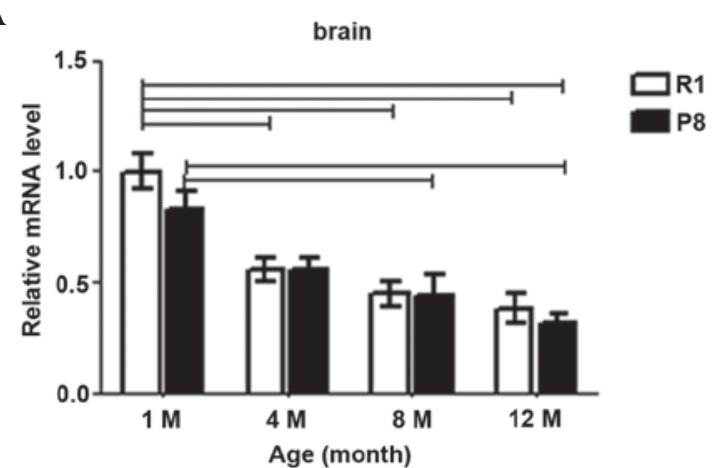

C

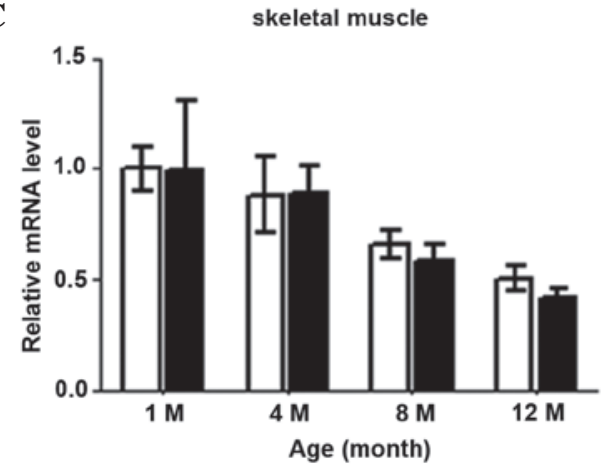

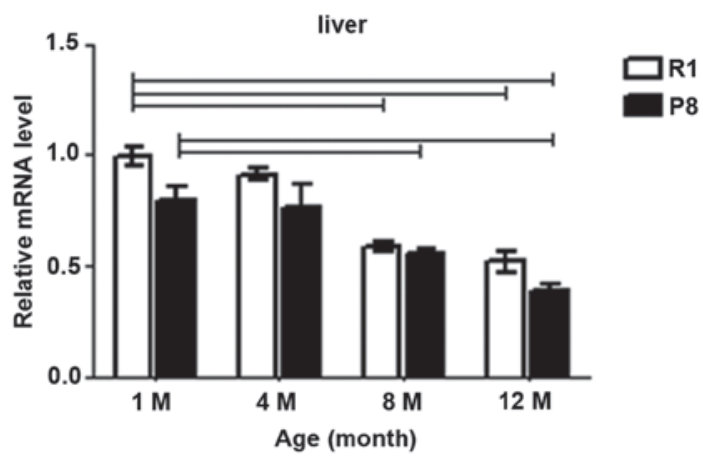

D

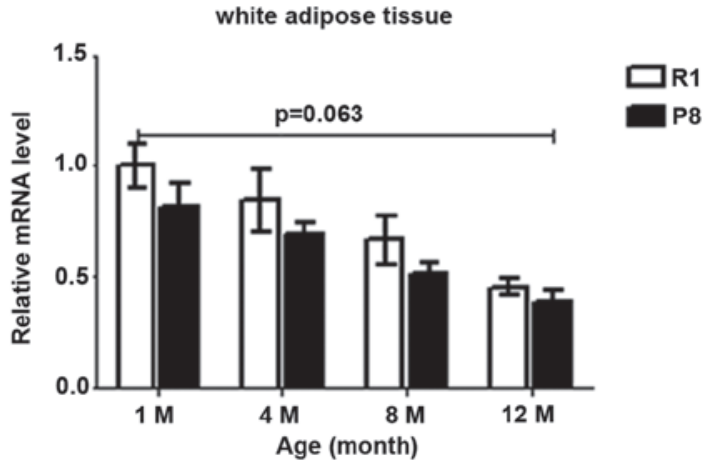

Figure 2. The changes of Sirt1 mRNA expression patterns in SAM-R1 and SAM-P8 in (A) brain, (B) liver, (C) skeletal muscle and (D) white adipose tissue with age. The values (mean \pm SEM) are expressed as a percentage relative to the SAM-R1 1-month-old group. n=3-6 animals/group. R1, SAM-R1; P8, SAM$\mathrm{P} 8 ; 1 \mathrm{M}, 4 \mathrm{M}, 8 \mathrm{M}$ and $12 \mathrm{M}, 1-, 4-, 8$ - and 12-month old groups, respectively. The dash indicates the difference is significant between the two groups $(\mathrm{P}<0.05)$. SAM, senescence-accelerated mouse; SAM-R1, senescence-accelerated mouse resistant 1; SAM-P8, senescence-accelerated mouse prone 8; Sirt1, sirtuin 1; SEM, standard error of the mean.

Age-associated changes in Sirt1 protein expression level in different tissues of SAM. The Sirt1 protein level was also detected in the tissues of the two strains at the various different ages. It also decreased with age in all of these four tissues in both strains (Fig. 3). With regard to the supposed maximal difference, compared with the SAM-R1 1-month old group, the Sirt1 protein level in the SAM-P8 12-month old group reduced to $31.8 \%$ in the brain (Fig. 3A and B), $65.6 \%$ in the liver (Fig. 3A and C), 65.9\% in skeletal muscle (Fig. 3A and D) and $74.5 \%$ in white adipose tissue (Fig. 3A and E). These differences were not always increased compared with those between 1- and 12-month old within the same strain of all the tissues. In the brain tissue, compared with the 1-month old mice, the Sirt1 protein level of the 12-month old group decreased to $54.4 \%$ in SAM-R1 and $35.3 \%$ in SAM-P8 (Fig. 3A and B). In the liver tissue, the expression decreased to $67.4 \%$ in SAM-R1 and $80.4 \%$ in SAM-P8 (Fig. 3A and C). In the skeletal muscle, it decreased to $74.4 \%$ in SAM-R1 and 65.4\% in SAM-P8 (Fig. 3A and D), and finally, in the white adipose tissue, the expression decreased to $91.9 \%$ in SAM-R1 and $59.2 \%(\mathrm{P}<0.05)$ in SAM-P8 (Fig. 3A and E).

As for the differences between SAM-P8 and SAM-R1 in the brain at the same age (Fig. 3A and B), the levels of SAM-P8 were lower than those of SAM-R1 at the same age and the differences increased with age: 90.0, 64.7, 62.2 and $58.4 \%$ (vs. SAM-R1) from 1- to 12-month old, respectively. While in the liver (Fig. 3A and C), there were almost no marked differences between the two strains, except that at 1-month old, the protein level of SAM-P8 was $18 \%$ lower than that of SAM-R1, although this difference was not significant. In the skeletal muscle (Fig. 3A and D), Sirt1 protein levels in SAM-P8 were also lower than in SAM-R1 at 8 -month (10\%) and 12-month old (7.6\%). In white adipose tissue (Fig. 3A and E), Sirt1 protein level in SAM-P8 was marginally higher (1.26-fold vs. SAM-R1) at 1-month old and lower (0.81-fold vs. SAM-R1) at 12-month old.

In the brain (Fig. 3A and B), the Sirt1 protein level declined evidently with age from 1-month old in both strains and more so in SAM-P8 (35.3\%) than in SAM-R1 (54.4\%): in SAM-R1, the protein expression reduced to 78.0, 60.7 and $54.4 \%(\mathrm{P}=0.051)$ and in SAM-P8, it reduced to 56.1, $42.0 \%(\mathrm{P}<0.05)$ and $35.3 \%$ $(\mathrm{P}<0.05)$ at $4-, 8$ - and 12-month old, respectively. In the liver (Fig. 3A and C), Sirt1 protein level in SAM-R1 reduced by $\sim 30 \%$ at 1-month old, while were relatively stable and only reduced moderately following 4-months: $72.9,69.1$ and $67.4 \%$ at 4-, 8- and 12-month old compared with 1-month old, respectively. In SAM-P8, the protein levels also only decreased slightly to 95.3, 90.2 and $80.4 \%$ at 4-, 8- and 12-month old compared with 1-month old, respectively. In the skeletal muscle (Fig. 3A and D), the change in Sirt1 protein level expression in SAM-R1 was similar to that in the liver: reduced from 1- to 4-month old, then maintained relatively stable: the levels were $75.1,73.5$ and 74.5\% in 4-, 8- and 12-month old group as at 1-month old group, respectively. While in SAM-P8, the Sirt1 protein level declined gradually from 1- to 8-month old, then remained stable from 8- to 12 -month old: $71.9 \%$ (4-month), $62.8 \%$ (8-month, $\mathrm{P}<0.05$ ) and $65.5 \%$ (12-month) compared with the 1-month old group. In the white adipose tissue (Fig. $3 \mathrm{~A}$ and E), the changing trends 
A

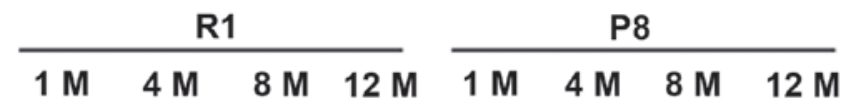
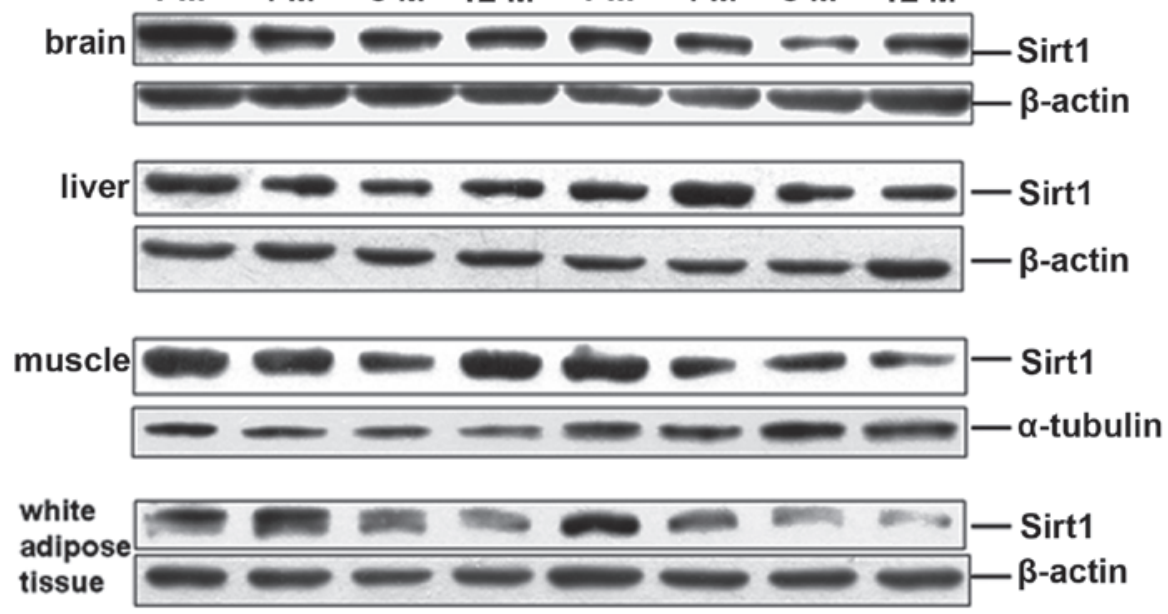

B

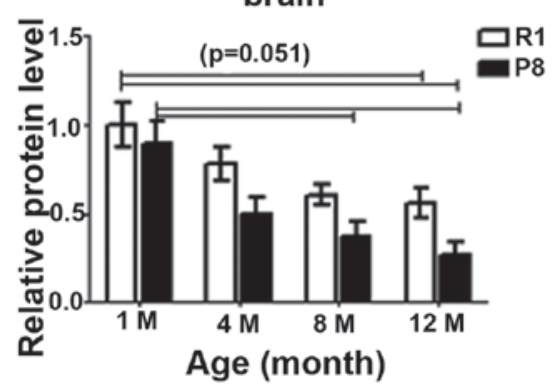

D

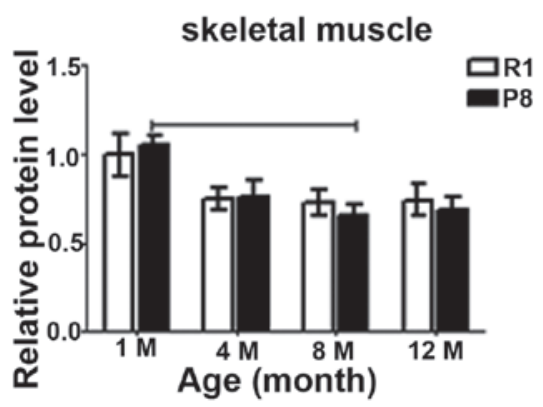

C

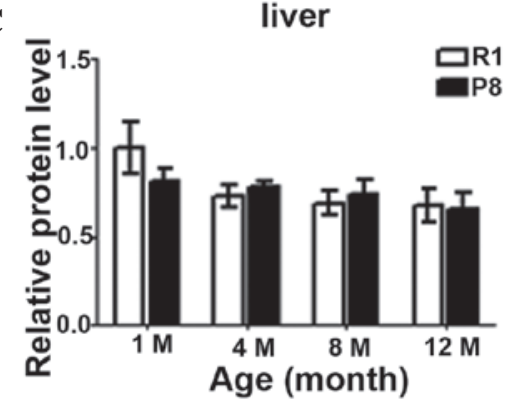

$\mathbf{E}$

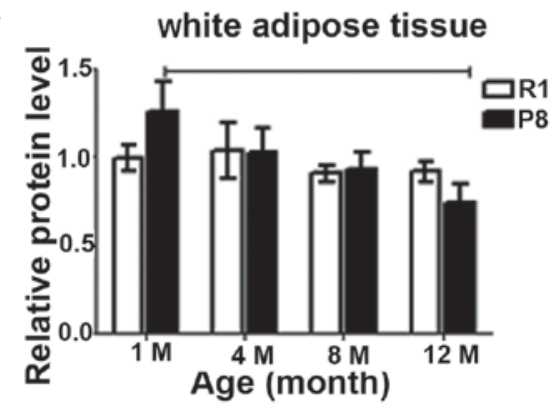

Figure 3. (A) Representative western blotting images and quantitative densitometric analysis of the changes of Sirt1 protein expression patterns in SAM-R1 and SAM-P in (B) brain, (C) liver, (D) skeletal muscle and (E) white adipose tissue with age. The values (mean \pm SEM) are expressed as a percentage relative to the SAM-R1 1-month-old group. n=3-6 animals/group. R1, SAM-R1; P8, SAM-P8; 1M, 4M, 8M and 12M, 1-, 4-, 8- and 12-month old groups, respectively. The dash indicates the difference is significant between the two groups $(\mathrm{P}<0.05)$. SAM, senescence-accelerated mouse; SAM-R1, senescence-accelerated mouse resistant 1; SAM-P8, senescence-accelerated mouse prone 8; Sirt1, sirtuin 1; SEM, standard error of the mean.

were weakly different from the above two tissues. In SAM-P8, the Sirt1 protein level gradually reduced to $81.7,74.3$ and $59.2 \%$ $(\mathrm{P}<0.05)$ at 4-, 8- and 12-month old compared with 1-month old, respectively; while in SAM-R1, it remained stable from 1- to 4-month (1.04-fold vs. 1-month) and from 8-month (0.91-fold vs. 1-month) to 12 -month (0.92-fold vs. 1-month) and reduced slightly from 4- to 8-month old (13.6\% as 4-month).

These data demonstrate that the Sirt1 protein level also decreased with age in all of the four tissues in both strains, however the degree at which the expression changed was not as marked as that observed at an mRNA level, except in the brain. The protein expression was reduced most in the brain than in the other three tissues. The differences between SAM-P8 and SAM-R1 at the same age were not evident in the liver, muscle and white adipose tissue, but the levels in SAM-P8 were lower than SAM-R1 in the brain.

\section{Discussion}

Aging in most species is associated with impaired adaptive and homeostatic mechanisms, leading to susceptibility to environmental or internal stresses with increasing rates of disease $(14,15)$. Aging is a multifactorial process characterized by a progressive decline in physiological function of bodily organs (16). Sirt1 is expressed throughout the body and has broad biological effects. Sirt1 has been demonstrated to have a crucial role in mammalian health and disease, and the regulation of metabolism, stress responses, genome stability and 
aging. To clarify systematically the natural changing pattern of Sirtl expression may facilitate our understanding of the natural aging process. It is possible that the level of Sirtl expression in each tissue may be a potential marker that reflects the degree of physiological function decline in a tissue with age.

In the present study, various different groups of animals which covered multi-facet parameters were established: i) age comparisons were selected between the young, maturing and old mice (1-, 4-, 8- and 12-month old); ii) both senescenceaccelerated prone and resistant strains, SAM-P8 and SAM-R1, which have different aging speeds were utilized; iii) four different tissues were examined, including brain, liver, skeletal muscle and white adipose tissue, which not only cover from low, moderate to high expression levels in all the 12 tissues that were originally scanned, but also include organs important in regulating metabolism and central nervous functioning; iv) finally, both transcriptional and translational Sirt1 expression levels were examined. These parameters contributed to the reliability of this systematic investigation of Sirt1 expression level with age.

In general, the results of the present study demonstrated that Sirtl expression decreased with age in all four tissues investigated in both strains at a transcriptional and translational level (Figs. 2 and 3). At a transcriptional level, the age-dependent reduction in Sirt1 expression was more marked in SAM-P8 than SAM-R1 in the liver and skeletal muscle, or similar to SAM-R1 in white adipose tissue and brain. In all the four tissues, the largest differences were evident between 1-month old SAM-R1 and 12-month old SAM-P8. At a translational level, the expression in SAM-P8 decreased more than SAM-R1 in skeletal muscle, white adipose tissue and brain. Furthermore, Sirt1 transcriptional level in SAM-P8 was $\sim 10-25 \%$ lower than in SAM-R1 at almost all the time points in these tissues following maturation. The protein level in SAM-P8 was also lower than in SAM-R1 at least at old age (12-month old) in all the four tissues. Previously, Sasaki et al (17) identified that the loss of Sirtl with age was accelerated in mice with accelerated aging but was not observed in long-lived growth hormone receptor knockout mice. In another study, diabetic animals demonstrated increased cellular senescence in renal glomerulus and retinal blood vessels, along with reduced Sirt1 mRNA expression in these tissues (18). Hyperglycemia accelerates aging-associated processes in the vascular endothelial cells and it appears such processes are mediated via the downregulation of Sirt1 (18). In the kidney, aging markedly decreased Sirt1 protein level in 24-month old animals as compared with 2- and 12-month old animals (16). These studies are in accordance with our findings and these data suggested there is a parallel correlation between Sirt1 expression level and the degree of aging, and the loss of Sirtl may be an important trigger for the aging process.

Loss of Sirtl with age has tissue specificity to a certain degree, particularly at a protein level. From 1- to 12-month old, Sirt1 mRNA decreased gradually in the liver, skeletal muscle and white adipose tissue to $50 \%$ (45.4-55.7\%), while in the brain it reduced to <40\%: $38.1 \%$ in SAM-P8 and $38.3 \%$ in SAM-R1. At a protein level, Sirtl also decreased gradually in the brain and reduced more in SAM-P8 (to 35.3\%) than in SAM-R1 (54.4\%). However, in the other three tissues, this effect was weaker than in the brain, particularly following maturation. In the brain, Sirt1 protein levels of SAM-P8 were lower than those of SAM-R1 at all the ages and the differences increased with age. These results suggested that the brain was the most sensitive to changes in Sirtl induced by age, particularly in the SAM-P8 model. Since the SAM model was established in 1981 (12), the substrain SAM-P8 mice have attracted notable attention in the gerontological research of dementia. This model is characterized by a marked increase in oxidative stress in the brain and the development of early learning and memory deficits (between 8 and 10 months) together with other characteristics similar to those observed in Alzheimer's disease (AD). Other characteristics of these mice include an altered circadian rhythm, reduced anxiety behavior (19), immune dysfunction late in their lifespan (20) and reduced life expectancy $(21,22)$. Sirt1 has been demonstrated to be involved in all of the above functions, including circadian rhythms $(23,24)$, immune responses $(25,26)$, oxidative response (27) and $\mathrm{AD}(28,29)$. As demonstrated in the results that the Sirtl expression level decreased more in SAM-P8 than in SAM-R1, it is possible that the loss of Sirt1 in SAM-P8 has an important role in the pathological characteristics of SAM-P8.

In conclusion, the present study systematically investigated age-associated changes in Sirtl expression pattern and found that Sirtl expression declined with age at the transcriptional and translational levels in liver, skeletal muscle, white adipose and brain tissues in two in vivo models of aging, SAM-P8 and SAM-R1. It was demonstrated that the Sirt1 level was lower in SAM-P8 than in SAM-R1, particularly at old age, and the most sensitive organ to this reduction was the brain.

\section{Acknowledgements}

The present study was supported by the Research Special Fund for Public Welfare Industry of Health to T.M.Z. (grant no. 201302008) and the National Natural Science Foundation of China to H.G. (grant no. 81300693)

\section{References}

1. Tissenbaum HA and Guarente L: Increased dosage of a sir-2 gene extends lifespan in Caenorhabditis elegans. Nature 410: 227-230, 2001.

2. Viswanathan M and Guarente L: Regulation of Caenorhabditis elegans lifespan by sir-2.1 transgenes. Nature 477: E1-E2, 2011.

3. Donmez G and Guarente L: Aging and disease: connections to sirtuins. Aging Cell 9: 285-290, 2010.

4. Guarente L: Franklin H. Epstein Lecture: Sirtuins, aging, and medicine. N Engl J Med 364: 2235-2244, 2011.

5. Imai S, Armstrong CM, Kaeberlein M and Guarente L: Transcriptional silencing and longevity protein Sir2 is an NAD-dependent histone deacetylase. Nature 403: 795-800, 2000.

6. Yamamoto H, Schoonjans K and Auwerx J: Sirtuin functions in health and disease. Mol Endocrinol 21: 1745-1755, 2007.

7. Banks AS, Kon N, Knight C, et al: SirT1 gain of function increases energy efficiency and prevents diabetes in mice. Cell Metab 8: 333-341, 2008.

8. Bordone L, Cohen D, Robinson A, et al: SIRT1 transgenic mice show phenotypes resembling calorie restriction. Aging Cell 6: 759-767, 2007.

9. Herranz D, Muñoz-Martin M, Cañamero M, et al: Sirt1 improves healthy ageing and protects from metabolic syndrome-associated cancer. Nat Commun 1: 3, 2010.

10. Cheng HL, Mostoslavsky R, Saito S, et al: Developmental defects and p53 hyperacetylation in Sir2 homolog (SIRT1)-deficient mice. Proc Natl Acad Sci USA 100: 10794-10799, 2003.

11. McBurney MW, Yang X, Jardine K, Bieman M, Th'ng J and Lemieux M: The absence of SIR2alpha protein has no effect on global gene silencing in mouse embryonic stem cells. Mol Cancer Res 1: 402-409, 2003. 
12. Takeda T, Hosokawa M, Takeshita S, et al: A new murine model of accelerated senescence. Mech Ageing Dev 17: 183-194, 1981.

13. Flood JF and Morley JE: Learning and memory in the SAMP8 mouse. Neurosci Biobehav Rev 22: 1-20, 1998.

14. Campbell KH and O'Hare AM: Kidney disease in the elderly: update on recent literature. Curr Opin Nephrol Hypertens 17: 298-303, 2008

15. O'Hare AM, Bertenthal D, Covinsky KE, et al: Mortality risk stratification in chronic kidney disease: one size for all ages? J Am Soc Nephrol 17: 846-853, 2006

16. Lim JH, Kim EN, Kim MY, et al: Age-associated molecular changes in the kidney in aged mice. Oxid Med Cell Longev 2012: 171383, 2012.

17. Sasaki T, Maier B, Bartke A and Scrable H: Progressive loss of SIRT1 with cell cycle withdrawal. Aging Cell 5: 413-422, 2006.

18. Mortuza R, Chen S, Feng B, Sen S and Chakrabarti S: High glucose induced alteration of SIRTs in endothelial cells causes rapid aging in a p300 and FOXO regulated pathway. PLoS One 8: e54514, 2013

19. Miyamoto M: Characteristics of age-related behavioral changes in senescence-accelerated mouse SAMP8 and SAMP10. Exp Gerontol 32: 139-148, 1997.

20. Powers DC, Morley JE and Flood JF: Age-related changes in LFA-1 expression, cell adhesion, and PHA-induced proliferation by lymphocytes from senescence-accelerated mouse (SAM)-P/8 and SAM-R/1 substrains. Cell Immunol 141: 444-456, 1992.

21. Morley JE: The SAMP8 mouse: a model of Alzheimer disease? Biogerontology 3: 57-60, 2002.
22. Morley JE, Armbrecht HJ, Farr SA and Kumar VB: The senescence accelerated mouse (SAMP8) as a model for oxidative stress and Alzheimer's disease. Biochim Biophys Acta 1822: 650-656, 2012

23. Asher G, Gatfield D, Stratmann M, et al: SIRT1 regulates circadian clock gene expression through PER2 deacetylation. Cell 134: 317-328, 2008 .

24. Belden WJ and Dunlap JC: SIRT1 is a circadian deacetylase for core clock components. Cell 134: 212-214, 2008.

25. Salminen A, Kauppinen A, Suuronen T and Kaarniranta K: SIRT1 longevity factor suppresses NF-kappaB -driven immune responses: regulation of aging via NF-kappaB acetylation? Bioessays 30: 939-942, 2008.

26. Sequeira J, Boily G, Bazinet S, et al: sirt1-null mice develop an autoimmune-like condition. Exp Cell Res 314: 3069-3074, 2008

27. Chong ZZ, Shang YC, Wang S and Maiese K: SIRT1: new avenues of discovery for disorders of oxidative stress. Expert Opin Ther Targets 16: 167-178, 2012.

28. Qin W, Yang T, Ho L, et al: Neuronal SIRT1 activation as a novel mechanism underlying the prevention of Alzheimer disease amyloid neuropathology by calorie restriction. J Biol Chem 281: 21745-21754, 2006

29. Kim D, Nguyen MD, Dobbin MM, et al: SIRT1 deacetylase protects against neurodegeneration in models for Alzheimer's disease and amyotrophic lateral sclerosis. EMBO J 26: 3169-3179, 2007. 